\title{
Applications and Limitations of Complexity Theory in Organization Theory and Strategy
}

David L. Levy

University of Massachusetts, Boston, Massachusetts

\section{INTRODUCTION}

Strategy concerns itself with the development and deployment of corporate resources in such a way as to compete more effectively in a particular industry. Despite the importance of strategy in the business literature, there is a paucity of understanding and consensus around foundational issues in the discipline. What exactly is the nature of those intangible competencies, capabilities, resources, and assets that enable one firm to succeed while another stumbles? What makes a particular competency difficult to duplicate or acquire? Must a firm's strategy fit the environment, or can a firm successfully shape its environment to suit its existing capabilities? The answers are elusive because strategy deals with highlevel abstractions concerning very complex systems. Business success and failure are outcomes of complex interactions between an organization and its changing environment, without simple cause and effect relationships; indeed, any patterns that we may discern may well prove ephemeral.

Industries evolve in a dynamic, path dependent manner over time as a result of complex interactions among firms, government, labor. consumers. financial institutions, and other elements of the environment. Not only does industry structure influence firm behavior, but firm behavior in turn can alter the structure of an industry and the contours of competition. Existing theoretical models, however, tend to assume relatively simple linear relationships without feedback. Many strategic frameworks attempt to classify firms and industries and to describe appropriate strategies for each class; examples include the Boston Consulting Group matrix for resource allocation and Bartlett's classification of international strategies (Bartlett and Ghoshal 1989). These models tend to be oversimplified and lack much explanatory or predictive value.

Complexity theory, which is the study of nonlinear dynamic systems. promises to be a useful conceptual framework that reconciles the essential unpredictability of industries with the emergence of distinctive patterns (Cartwright 1991). Although the theory was originally developed in the context of physical and biological sciences. Butler (1990), Kiel and Elliott (1996). Merry (1995), and Radzicki (1990), among others, have noted that so- 
cial, ecological, and economic systems also tend to be characterized by nonlinear relationships and complex interactions that evolve dynamically over time. This recognition has led to a surge of interest in applying complexity theory to a number of fields. including medicine (Goldberger et al. 1990). international relations (Mayer-Kress and Grossman 1989), and economics (Baumol and Benhabib 1989; Kelsey 1988; Medio 1991; Mirowski 1990). ' Some authors appear overcome with evangelical zeal in their enthusiasm for the new science, invoking mystical and biblical overtones. Merry (1995:13), for example, writes, "Deep chaos is a natural. unescapable essential stage in the transformation of all life forms. Out of chaos come forth the fertile variety of forms of existence and life in this universe."

During the 1990s, there was an explosion of interest in complexity as it relates to organizations and strategy. There are several major themes in complexity theory that explain its appeal to scholars of organizations. Understanding the complexity of organizations and their environments has been a long-standing concern of organization theory. Simon (1962; 1964) viewed the analysis of complexity and the application of analytical and computer tools to study complex systems as laying the groundwork for a unified theory of management. Systems theory (Katz and Kahn 1966; Thompson 1967) also promised a theoretical synthesis that could integrate multiple levels and perspectives. In this sense, complexity theory should be seen as a continuation of earlier efforts rather than a complete paradigm shift.

Nevertheless, complexity theory offers a number of new insights, analytical methods, and conceptual frameworks that have excited many scholars of management in recent years. It suggests that simple deterministic functions can give rise to highly complex and often unpredictable behavior, and yet this complexity can still exhibit surprising order and patterns. It may offer a synthesis of two competing perspectives on how organizations adapt to their environments, organizational adaptation and population ecology. Most tantalizing, perhaps, is the promise that complexity theory will lead us to understand how systems can learn more effectively and spontaneously self-organize into more structured and sophisticated forms that are better adapted to their environments. Although these findings are as yet tentative and confined to computer simulations of simplified networks, management consultants are rapidly springing up, claiming to be able to apply complexity principles to bring organizations to "the edge of chaos," enhancing creativity, learning, and adaptation.

This chapter introduces readers to complexity theory and discusses its relevance to the social sciences in general and to organizational theory and strategy in particular. It is useful to begin with a detailed but accessible presentation of the basic theory of chaos and complexity in order for readers to understand the genesis of the field and the terminology used. In light of some of the loftier claims about the complexity revolution, this background is particularly important in enabling readers to judge for themselves its potential application to organizations and strategy.

Complexity theory is the study of complex, nonlinear, dynamic systems with feedback effects. For the sake of clarity, chaos theory is here distinguished from network theory, and the term "complexity" is used as an umbrella concept that includes both chaos and networks. Chaos theory is concerned with systems in which the recursive application of nonlinear deterministic functions can give rise to apparent random behavior and subtle patterns. Network analysis, with which complexity theory has been more closely associated in the 1990s, investigates the properties of networks of nodes where the state of each node is a function of its connections to other nodes. The relevance to brains, as neural networks; to 
organizations, as networks of departments and people; and to industries, as networks of firms should be immediately apparent. Although many applications of complexity entail some hybrid of chaos and network theory, each is described separately here for the sake of simplicity and clarity.

\section{AN INTRODUCTION TO CHAOS THEORY}

Chaos theory was pioneered by Lorenz (1963), who was studying the dynamics of turbulent flow in fluids. ${ }^{2}$ Although we all recognize the swirls and vortices that characterize turbulent flow, the complexities of turbulent flow have confounded mathematicians for years. A similar problem arises when trying to calculate the path of an object in the gravitational pull of two or more bodies. Although we can use simple Newtonian equations to predict the orbits of planets around the sun with a high degree of accuracy, the mathematics involved in the case of two or more "suns" become intractable. The problem can be illustrated on a terrestrial level by observing the motion of a simple toy, a metal ball suspended over two or more magnets. The ball traces a series of patterns that never exactly repeat themselves and yet are not totally random.

The paradox here is that the motion of the metal ball is driven by the same Newtonian equations as the well understood case of a single gravitational attractor. If we knew precisely the original location, speed, and direction of the ball, we ought to be able to predict its path with a reasonable degree of accuracy. How is it that deterministic systems can give rise to unpredictability? The explanation is that tiny variations in the motion of the ball are magnified every time it swings by one of the magnets. It is a combination of this divergence and the repeated interactions that gives rise to "chaotic" behavior. Mathematically, chaotic systems are represented by differential equations that cannot be solved, so that we are unable to calculate directly the state of the system at a specific future time $t$; computer modeling and simulation techniques need to be employed to follow the path of such a system.

At the limit, chaotic systems appear truly random. A toss of a coin and the roll of a die are, in theory, deterministic systems but yield more or less random outcomes. Not only is it impossible to toss a coin twice in exactly the same way, but on each toss the coin is subject to slightly different air currents, themselves a result of turbulent air flow (Ford 1983; Stewart 1989). To overcome the problem of intractable differential equations, researchers usually model systems as discrete difference equations, which specify what the state of the system will be at time $t+1$ given the state of the system at time $t$. Computer simulations can then be used to investigate how the system evolves over time.

One of the major achievements of chaos theory is its ability to demonstrate how a simple set of deterministic relationships can produce patterned yet unpredictable outcomes. Chaotic systems never return to the same exact state, yet the outcomes are bounded and create patterns that embody mathematical constants (Feigenbaum 1983). It is the promise of finding a fundamental order and structure behind complex phenomena, both physical and social, that explains much of the great excitement and interest chaos theory has generated in so many fields.

The logistic difference equation is frequently used to illustrate basic concepts of chaos theory and its application to ecological models of population fluctuations. This equation has the form

$$
P_{t+1}=P_{t}^{*} R *\left(1-P_{t}\right)
$$


$P$, a fraction between 0 and 1 , represents the population level as a proportion of the maximum carrying capacity of the environment, $R$ is the growth rate from one cycle to the next, and population growth is constrained by the factor $1-P_{t}$, which can be understood as a resource constraint. When the parameter $R$ is less than 3, recursive application of this equation quickly leads to a steady state from any starting value of $P$ between 0 and 1 . As $R$ is increased past 3 , the system suddenly starts to exhibit periodic behavior, oscillating between two values. As $R$ is increased further, the period doubles to 4, then doubles again. These period doublings, or bifurcations, come faster and faster until, as $R$ approaches 3.57, the system suddenly turns chaotic; the system never returns to the same precise value twice. As $R$ is increased further, the system may suddenly revert to a periodic regime; for a brief interval around $R=3.74$, the system has a simple 5-period cycle. As $R$ is increased again, we see the familiar period doubling and chaos quickly returns.

Even this simplest of equations possesses two properties that are of significance for social scientists. The first is that the system is highly sensitive to initial conditions. Suppose we run the system twice starting with two very slightly different values of $P$. Within a few iterations, the system diverges as a result of the repetitive application of a nonlinear equation. This is the cause of the "butterfly effect," a term attributed to Lorenz, who, while working on nonlinear weather models, remarked that a butterfly flapping its wings in Mexico might alter the weather in Texas. The second property of such systems, closely related to the first, is that their long-run behavior cannot be predicted; the only way to know the value of the system at time $t=100$ is to run the system for 100 periods.

Much of the interest in chaotic systems lies, paradoxically, in their underlying patterns of structure and order, even when they are in the chaotic state. This is perhaps best illustrated by using the concept of attractors. Imagine a nonlinear dynamic system with three variables, for example, a simple weather model using temperature, air pressure, and humidity. We could plot the values of these variables on a three-dimensional graph at successive points in time; these points would map out the phase space of the system. Depending on the system's parameters, it might tend toward a stable equilibrium; from any starting point, the graph would curve into a single point, the attractor. A periodic system might trace out a repetitive orbit; the term attractor, strictly speaking, defines the set of points in phase space, though it is commonly used to mean the imaginary center of this orbit. Note that the system never reaches the point of attraction, however, just as the earth does not crash into the sun.

Chaotic systems exhibit strange attractors, elliptical or perhaps torus shaped orbits that, though never repeating themselves precisely, appear constrained to trace a particular pattern in phase space. The weather is a good example of a chaotic system, as precise conditions are never repeated in any one location, let alone around the world, yet predictable patterns and limits can be observed. ${ }^{3}$ Chaotic attractors may be relatively homeostatic, meaning they can remain quite stable as system parameters are changed, but the system can also flip suddenly to a very different attractor when a parameter passes a particular threshold level. ${ }^{4}$ Because of path dependency, the system may not necessarily return to its prior state if the parameter causing the change is pushed back to its former level.

Despite the simplicity of the underlying deterministic equations, chaotic systems are capable of sudden, dramatic changes. One of the important insights of chaos theory is that dramatic change can be endogenous to the system; the collapse of a population of a particular species may not be due to some extraterrestrial meteoric impact, but rather the result of the dynamics of the system itself. Similarly, a collapse in the stock market may be due to the positive feedback mechanisms associated with investor confidence. This raises the 
issue of what is considered endogenous and what is exogenous; to a certain extent, this depends on some arbitrary definition of the boundaries of a system. A model of a national economy might regard economic or military measures by foreign governments as exogenous, but a larger model that included other countries and political systems could attempt to endogenize such variables. A general feature of such systems is that the degree of change in any period in a chaotic system tends to follow a power law (Bak and Chen 1991); small changes are relatively frequent, and the probability of large changes declines more than proportionally to the size of the change.

\section{NETWORK THEORY}

Much of the groundwork of network theory was laid by theoretical biologists such as Stuart Kauffman $(1993 ; 1995)$ and Christopher Langton $(1989) .{ }^{5}$ Kauffman was fascinated by the concept of self-organization in soups of simple proteins and enzymes from which life might emerge. Understanding that chance and natural selection were too slow and cumbersome to yield sophisticated life-forms out of the basic building blocks of organic chemistry, he pioneered the use of computers to simulate networks of organic chemicals. Kauffman hypothesized that the precursor to living cells might be an autocatalytic set of proteins and enzymes, which maintains and reproduces itself through a cycle of chemical reactions in which each organic molecule is the output of one reaction and the input into another. In Kauffman's computer models of this chemical soup, $N$ is the number of nodes, each representing one chemical, and each of which can only be in one of two states, on or off, representing whether or not the chemical is being synthesized. Each node is switched on and off by its connections to other nodes, with the parameter $K$ representing the number of other nodes each is connected to. Once the system is switched on from some starting point, the logical connections among the nodes cause some of them to switch on and off in successive periods (nodes can be thought of bulbs switching on and off).

By studying large numbers of these networks, Kauffman found some recurring patterns. These networks have attractors, in the sense that from any random starting distribution of nodes being on and off, the system is attracted to and settles into a periodic cycle. When $K$ is set equal to $N$, meaning every node is connected to every other, there are $N / e$ attractors (where $e$ is the natural logarithm constant 2.71828), and the average length of state cycles is the square root of the total number of possible states, or $2^{N / 2}$. With even modestly sized networks, say $N=100$, the length of state cycles is so long that the nodes appear to flash on and off randomly; if the network took a millionth of a second to pass from state to state, the state cycle would still be hundreds of billions of years. Any small change to the system, say tlipping the state of one node or changing a network connection, completely alters the attractor basins and the future evolution of the network. Network theorists term such networks chaotic and see little useful order in them.

These networks can become overly stable if $K$ is too low and the $P$ bias parameter is close to 1 or $0 .{ }^{6}$ Such sparsely connected systems are very rigid and uninteresting; they tend to have very short state cycles, often only a single state, and vast numbers of small basins of attraction; the system essentially freezes up very quickly. A small perturbation to the system causes virtually no change.

Kauffman's major discovery was that such networks can become highly ordered, yet flexible and adaptive, when the system is tuned by using the $K$ and $P$ parameters. In a system with $K=2$, the average length of state cycles is approximately the square root of $N$, 
the number of nodes. If $N=100,000$, the number of genes in the human genome, the average period of the system is $\sqrt{100,000}$ or about 317 states; such systems, on average, also contain only about $N$ basins of attraction, meaning that from near-infinite $\left(2^{N}\right)$ potential starting positions, the system settles into relatively few patterns of behavior. This is the phenomenon of self-organization, or what Kauffman called "order for free." When the system has settled into a particular state cycle attractor, large numbers of nodes are permanently switched on, and these form an interconnected network. Kauffman hypothesized that this process underlies the emergence of autocatalytic sets of proteins; nature does not rely just on random evolutionary search across the vast state space of such systems. If each node in a network is understood to represent one human gene. then a particular attractor is related to a specific group of genes switched on, and in turn this can be associated with a particular human cell type. There are approximately 256 different human cell types, close to the number of attractors in a 100,000-node system.

Systems in this ordered, yet flexible state are said to be "on the edge of chaos." They are quite homeostatic, meaning that flipping any one node from on to off, or changing the nature of one connection, has little effect on the basins of attraction. Langton (1989) hypothesized that systems in this transition state between frozen order and random chaos are capable of complex forms of coordination, as perturbations in one site have limited but patterned effects across the system. The extent of change appears to follow a power law, as in chaos theory; occasionally, small perturbations might have much larger systemic effects. These systems are also adaptive, in the sense that if changes in the environment cause small perturbations to the system, it will reconfigure itself while retaining most of its previous structure.

\section{A. Fitness Landscapes}

A related application of network theory is the concept of evolution over fitness landscapes. Imagine that each node in a network represents one gene (say, for height or eye color), and each combination of genes is associated with the organism's prospects for success in its environment-its fitness level. The fitness landscape can be represented as $N$-dimensional terrain, where the height of the landscape represents the organism's fitness. $K$ here represents the extent to which the contribution of one gene to fitness depends on other genes; the usefulness of wings, for example, is highly correlated with possession of light bone structure. The higher $K$, the more genes interact and constrain each other, with the result that the fitness landscape is more rugged with more local peaks.

Organisms can move across this landscape, searching for higher levels of fitness, through processes of mutation and natural selection. An organism might mutate one gene at a time, at random, and test whether it helps it climb the landscape. Through natural selection, organisms can spawn offspring with random mutations, with those at a higher level of fitness having a greater chance of survival. Both of these processes lead organisms to climb local peaks, and natural selection then favors those at more distant higher peaks. This search process is aided by two important features of fitness landscapes with a moderate amount of ruggedness. ${ }^{7}$ High peaks are associated with large basins of attraction, just as high mountains tend to cover large geographic areas. As a result, if a population starts off randomly distributed over the landscape, more organisms will end up on higher peaks than on lower ones. Second, the correlation among genes means that the space between two high regions is likely to contain even higher terrain; combining genetic patterns from two relatively fit parents is thus likely to lead to even fitter offspring. 
Fitness landscapes are not necessarily stable over time. The evolution of one organism, say, a predator, deforms the fitness landscape for its potential prey, raising the importance of characteristics enabling it to escape or evade detection. This form of coevolution has been observed in many species sharing the same ecosystem. The implication is that organisms are constantly searching for fitness peaks in a shifting landscape, and so never reach an optimum or equilibrium point.

Computer modeling of these fitness landscapes illustrates a number of methods for improving the search process. One approach is occasionally to test a random distant point rather than continue to search the local neighborhood. The rate of progress in any particular locale slows quickly, as fewer changes that can lead further up the fitness slope are available. Less intuitively, the network of genes can be broken down into clusters, or patches, each of which can be optimized separately, holding the boundaries constant (Kauffman, 1995). This is equivalent to optimizing for subsystems, such as eyes, hands, and ears, rather than trying to optimize the whole body at once. The boundary constraints can then be relaxed, and the process repeated, taking into account the new information from other subsystems. The subsystems effectively coevolve, and the overall system performs better than if the search process takes the whole system as one unit. A third mechanism entails "jolting" the system with occasional disruptive shocks, a process likened to annealing metal. To understand this, it is useful to think of the fitness landscape inverted, so that peaks are now deep valleys. The search process drives the population of organisms into the valleys, but some become "stuck" in relatively small, shallow valleys. Now imagine giving the whole landscape a gentle shake, moving all the organisms a little away from the local minima they have settled into. Those in very shallow minima will be shaken out and are likely to fall into larger valleys, whereas those already in large valleys will not be moved enough to escape them. If the process is repeated with successively larger jolts, some of the organisms are likely to find the deepest valleys quite quickly.

\section{COMPARING CHAOS AND COMPLEXITY THEORY}

Both chaos and complexity theory attempt to reconcile the essential unpredictability of nonlinear dynamic systems with a sense of underlying order and structure. There are, however, some significant differences between the two approaches. Chaos theory searches for a small number of deterministic mathematical functions driving a system; in population models, for example, these functions might represent the fluctuations in the numbers of a species. Network theory is less concerned with underlying simplicity; it tends to rely on brute computing power to model large numbers of nodes connected by simple logical rules. Network theory is more interested in the emergent order and patterns in complex systems than in an attempt to find a simple mathematical "engine" in the system. Network models often try to capture the essence of interaction among the many agents in a system, whereas chaos theory generally attempts to model some resultant outcome, such as prices or investment.

The nature of "chaos" is understood differently in each approach. In chaos theory, it is the chaotic state that is interesting and that contains underlying order in the form of strange attractors and fractals. In network theory, by contrast, the chaotic regime is generally considered to be too random and disorderly to have much application. Network theory is most interested in systems that are in the ordered regime but approaching the phase transition to chaos; this "edge of chaos" condition is thought by some of its more ardent proponents to hold the secret of everything from learning in the brain to the evolution of life. 


\section{COMPLEXITY THEORY AND THE SOCIAL SCIENCES}

A basic understanding of complexity theory enables us to consider its application to the social sciences and to organization theory and business strategy. Proponents of complexity theory enthusiastically see signs of it everywhere, pointing to the ubiquity of complex, dynamic systems in the social world. That the world of economics and politics is complex and dynamic there is no doubt; less clear is the extent to which traditional linear models and approximations are inadequate, or how well complexity theory can fill the gaps.

Economic and social systems, from single firms to the global economy, all involve a multitude of actors interacting in complex ways. These interactions are iterative, in the sense that the outcomes of one period become the starting point of the next, giving rise to path dependency. The relationship between complexity and systems theory becomes clear when we consider that nodes in a system can be neurons in a brain, people in a department, departments in an organization, and organizations in a society. Attempts to model social and economic systems typically involve a hybrid approach using elements of chaos and network theory. A model might define social and economic actors as the nodes in a system and relate these nodes to each other in terms of variables such as prices, incomes, or decisions to invest. Given the state of the system at time $t$, we can calculate the state of the system at $t+1$, after going through all the connections and feedback loops. Radzicki (1990:59) calls this approach "an institutional dynamics model."

Advocates of complexity theory often claim that traditional approaches to social science have been overtaken by the increasing complexity of social and economic life, driven by swift developments in technology, communication, and transportation (Merry 1995). According to Brown and Eisenhardt (1997:3), "Many paradigms in organizational and strategic thinking, such as transaction cost economics, agency theory, and organization ecology, in which organizations are assumed to be static or nearly so... were developed in the 1970s : /hen speed and flexibility were less relevant to organizational success than they are for contemporary firms." It should be noted, however, that the application of complexity in the natural sciences does not depend on some absolute judgment of the rapidity of change; Kauffman considers it remarkable that the earth has evolved such diversity of life in just a few hundred million years. Critics such as Shackley, Wynne, and Waterton (1996) point out that social and economic life has always been complex, and that societies in every era have considered themselves to be subject to unusual levels of change and stress.

The complexity paradigm rejects some key assumptions of traditional neoclassical economics, such as perfect information, diminishing returns, and the implicit existence of a single rational agent acting on behalf of an organization to maximize some objective function. The ontology of complexity insists that the rational actor approach neglects key features of dynamic systems with multiple actors and feedback effects; a true understanding of these systems can only be gained by studying the complexity that is their very essence. Complexity thus also rejects a reductionist epistemology built upon simplifying assumptions and algebraic modeling of maximizing behavior by omniscient incarnations of the organization. More pertinent is the behavioral and administrative approach to organization theory pioneered by Simon (1957) and Cyert and March (1963), which recognizes that organizations comprise networks of people with bounded rationality. As a result, organizations are expected to "satisfice" rather than optimize, attend to subgoals sequentially rather than maximize a single objective function, adopt standard operating procedures to cope with routine situations, and search for improved structures and processes only when stimulated to do so. 
Scholars attempting to apply complexity concepts to organization and management studies have adopted two broad methodological approaches. One stream of research, firmly located in the traditional scientific paradigm, attempts to model complex systems by using sophisticated computer simulations and mathematical investigation. Their hope is that well-specified models can capture the patterns of behavior of a system and its responses to parameter changes even if they cannot predict the precise state of a system at some future time. Others have rejected this search for rigor and assert that understanding complex systems requires the application of nonpositivist methods, such as ethnographies, longitudinal studies, and metaphorical analogies, which employ inductive approaches to find patterns and meaning (Stacey 1995).

The advent of complexity theory is often associated with the much heralded end of modernism, with its accentuation of deterministic positivism, and the rise of the postmodern paradigm, which embraces the complexity, diversity, and contingency of experience. ${ }^{8}$ It should be remembered, however, that chaos theory does concern itself with the search for underlying order, and many of its applications, particularly in economics, are clearly looking for predictive power. Shackley, Wynne, and Waterton (1996) have cautioned that complexity theory, far from representing a break with the tradition of instrumental-technical rationality, risks becoming a search for more sophisticated tools of social control.

To understand the relevance of complexity to strategy, we need to conceptualize industries as dynamic, nonlinear systems. As Stacey (1995:480) puts it, "Nonlinearity and positive feedback loops are fundamental properties of organizational life." Much of the industrial organization aspect of strategy literature concerns itself with how firms interact with each other and with other actors in their environment, such as consumers, labor, the government, and financial institutions. These interactions are strategic in the sense that decisions by one actor take into account and anticipate reactions by others, and thus reflect a recognition of interdependence. Although interfirm behavior has been modeled formally in economics and business strategy by using game theory (Camerer 1991; Ghemawat 1997), these models tend to presume the emergence of equilibrium and do not adequately reflect industry dynamics. As Porter (1990) emphasizes, the evolution of industries is dynamic and path dependent: Corporate (and country-level) capabilities acquired during previous competitive episodes shape the context for future competitive battles. Moreover, the accumulation of competitive advantage can be self-reinforcing, through processes related to standard setting and economies of scale, suggesting important sources of nonlinearity.

One central concern of business strategy is the "fit" between organizations and their environments. Whether the ecological analogy is explicit or implicit, it is generally assumed that businesses will perform better when they deploy their assets in a manner that is appropriate to the environment. There has been substantial debate concerning how organizations come to achieve this fit (Hannan and Freeman 1984; Haveman 1992; Singh et al. 1986). The contingency view holds that organizations are capable of adapting and adjusting as their environments change, whereas the population ecology view sees organizations as quite inflexible, as a result of inertia and the costs of change. In the population ecology view, which borrows concepts and methodologies a little too freely from natural biology and ecology, natural selection drives populations to adapt to their environments. Our discussion of networks and fitness landscapes suggests that complexity theory has a great deal to offer in this area and that both adaptation and natural selection play a role in achieving fit. The fit is never perfect, however, as landscapes shift over time as result of coevolution of firms, their competitors, and their broader environments. A particular problem with the usual landscape models is that they assume that movement across the landscape is "free," 
whereas a fundamental principle of strategy is that valuable corporate assets, resources, structures, and capabilities, although not immutable, are costly to develop and change.

Despite its attractions, the application of complexity theory to the social sciences is still in its infancy and some think that expectations are too high (Baumol and Benhabib 1989). Although many economic and social processes may resemble the patterns generated by simple nonlinear systems, that does not mean that we can easily model and forecast these phenomena; it is almost impossible to take a set of data and determine the system of relationships that generates it (Butler 1990). In fact, there is considerable debate in the economics and finance literature about how one tests a data series to determine whether it is chaotic or simply subject to random influences (Brock and Malliaris 1989; Hsieh 1991). Moreover, it is important to recognize that many systems are not chaotic and that within certain parameters, linear approximations are good enough.

The application of complexity theory to social science has been constrained by the fact of its development in relation to physical and natural systems, without taking into account the fundamental differences between these and social systems. In the social world, outcomes often reflect very complex underlying relationships that include the interaction of several potentially chaotic systems; carbon emissions into the atmosphere, for example, are influenced by the interaction of economic, political, and natural systems. ${ }^{9}$ Chaos theory reflects a possibly futile search for a simple underlying mathematical engine driving such a system, and network theory tends to oversimplify the relationships among nodes.

Social and physical systems also differ in the source of unpredictability. In the physical world, unpredictability arises as a result of repeated iterations in nonlinear systems and our inability to define starting conditions with infinite precision. In the social world, we encounter difficulties in defining, let alone measuring, the conceptual constructs we take to be the variables at work in a system, and we lack the rigorous foundations of natural sciences in guiding our efforts to specify the structure of the system and the network connections.

A final difference is that physical systems are shaped by unchanging natural laws, whereas social systems are subject to intervention by cognizant agents, whose behavior is essentially unpredictable at the individual level. Investigations of economic time series by chaos theorists have usually assumed that relationships among economic actors are fixed over time. In reality, methods of macroeconomic management have changed from the use of the gold standard to Keynesian demand management and, later, to monetarist controls. Human agency can alter the parameters and very structures of social systems; indeed, one of the main purposes of management is to limit the gyrations of chaotic systems, reduce their sensitivity to external shocks, and, in the case of Demming's lean management systems (Womack and Jones 1990), ensure that behavior is nonchaotic by reducing variability throughout the system.

\section{APPLICATIONS OF COMPLEXITY THEORY}

\section{A. Modeling Economic and Social Systems}

Efforts to model features of complex economic and social systems generally entail a hybrid approach that combines the nonlinear iterative relationships of chaos theory with an effort to represent networks of agents, such as economic sectors, firms, consumers, and government. Radzicki (1990), in one example of this approach, developed a model to illustrate the nature of competition between two political parties. Positive feedback mechanisms lead 
one party (hawks or doves) to dominate for a while, but negative feedback eventually causes a "revolution" and the opposing party gains power. Mosekilde and Rasmussen (1986) have modeled long waves or cycles of economic development by using a similar approach. Some scholars have attempted to calibrate their models by using real economic data. Allen, Clark, and Perez-Trajo (1992) developed a planning model for developing countries, calibrated with data from the Senegalese economy, which seeks to capture the interdependencies among households, producers, and ecological variables, nodes that are linked by flows of information, people, resources, and money around the system.

Instead of relying on simulated agents with predetermined response functions, there is some interest in experimental approaches using real human subjects to act as agents in a simulated economic system. Sterman (1989), for example, used human subjects to play the role of managers of the capital producing sector. The subjects decided how much capital to order each period to minimize costs and balance supply and demand for each sector; demand was driven endogenously by the combined decisions of the subjects. As a result of time lags and feedback, the managers chronically overshoot, as capital stock cannot be increased fast enough in an upswing nor reduced during a downturn in demand. Sterman used the results to estimate the decision rules used by managers and demonstrated that realistic system parameters would give rise to chaotic outcomes.

\section{B. Models of Firms and Industries}

Recognizing that competition in real industries does not take place under the conditions assumed by economists, Arthur $(1989 ; 1996)$ has developed models of business competition that strive to incorporate some of the awkward realities of industrial competition in the real world. He has demonstrated, for example, that when two or more technologies compete for a market of potential adopters, the effect of standards, learning, and increasing returns to scale can quickly push the market to converge, or "lock-in" on a particular solution, which is not necessarily the superior technology nor the least-cost method. The dominance of Microsoft's Windows software, the QWERTY keyboard, and internal combustion engines are among the many examples Arthur sees as following this process. Under these conditions, a small initial advantage or even a chance event leads to the triumph of one technology. As time goes on, this technology is slowly refined, making it even harder for radical alternatives to be considered. With these types of market imperfections, we are likely to see a "winner-take-all" scenario rather than a stable equilibrium in which multiple firms can coexist.

Levinthal (1997) has applied Kauffman's network models directly to the issue of businesses adapting to their environment. Using Kauffman's NK network simulations, Levinthai has demonstrated how local search in a rugged landscape gives rise to a diversity of organizational forms, whereas natural selection gradually leads one form to dominate. This approach integrates adaptation at the organizational level with the population ecology view as mechanisms for generating fit between organizations and their environments. The tendency to become stuck on local fitness peaks suggests the importance of path dependency, as firms are always searching for higher peaks on a shifting landscape rather than reaching a global optimum. Levinthal's simulations also demonstrated that tightly coupled systems, in which each characteristic of the firm is related to many others, find it harder to adjust to changes in their environment. This is because their landscapes are too rugged with many local peaks, so that local search provides little information concerning the location of more distant but higher fitness terrain. Levinthal relates this find- 
ing to Henderson and Clark's (1990) observation that companies find it difficult to adapt to technological change that affects the architecture of systems, meaning the basic structural configuration of components. When the system is tightly coupled technologically, a change to any one component may require changes in the others and in the way the entire system is configured.

Levinthal notes that the nature of technological change (Abernathy and Clark 1985; Anderson and Tushman 1990; Henderson and Clark 1990) bears an interesting resemblance to the uneven process of species diversification in the natural environment described by Kauffman. Periods of relative stability are punctuated by brief phases of ferment during which many new forms emerge, a high rate of extinction occurs, and structural changes ensue. Early in the life of a new industry, we often witness a multiplicity of competing technologies, produced by local search and adaptation. In the automobile industry at the beginning of the twentieth century, manufacturers competed with electric, steam, and internal combustion engines. Population selection processes, reinforced by the feedback mechanisms described by Arthur, lead to the emergence of a single dominant form. A major disruption or upheaval is required before the dominance of internal combustion engines can be challenged; this may be about to occur over the next couple of decades as a result of the threat of climate change and restrictions on fossil fuel use.

Brown and Eisenhardt (1997), in a study of the computer industry, have challenged this model of punctuated equilibrium and maintain that complexity is also compatible with rapid, continuous change; those companies capable of continuous change, such as HewlettPackard, are among the most successful. Brown and Eisenhardt argue that the ability to sustain continuous change, rather than a particular static configuration of resources and assets, is an important core capability of great strategic value. This capability was associated with organizations that combined limited structure with extensive communication, a state evocative of "edge of chaos." Moreover, they "looked to the future using a variety of lowcost probes" (p. 7), suggesting an effective combination of flexibility and preparation for multiple scenarios.

Levy (1994) has developed a supply chain model calibrated on the data for a single company. The model stimulates the system's responses to perturbations caused by changing levels of demand, late shipments from vendors, or internal manufacturing problems. It was found that management planned operations on the basis of a reasonably stable supply chain and failed to anticipate how interactions along the chain caused wild fluctuations in inventory levels and orders to vendors. Levy suggested that the model could be utilized to identify and reduce sources of instability and establish inventory buffers sufficiently high to weaken the coupling among components of the supply chain. This would tune the supply chain to prevent perturbations from cascading and amplifying through the system, thereby avoiding a chaotic state.

\section{IMPLICATIONS OF COMPLEXITY THEORY FOR STRATEGY}

\section{A. Long-Term Planning Is Impossible}

Chaos theory has demonstrated how small disturbances multiply over time because of nonlinear relationships and feedback effects. As a result, such systems are extremely sensitive to initial conditions, making their future states appear random. Networks, even when in the ordered regime, are subject to perturbations from external influences, which sometimes cause substantial, though unpredictable reconfigurations. 
Forecasting is particularly difficult in systems that never approach a stable equilibrium state. The traditional approach to understanding the influence of industry structure on firm behavior and competitive outcomes has been derived from microeconomics, with its emphasis on comparative statics and equilibrium. Even the most complex game theoretic models are only considered useful if they predict an equilibrium outcome. By contrast, chaotic systems do not reach a stable equilibrium; indeed, they can never pass through the same exact state more than once. Organizations wander the shifting terrain of fitness landscapes on infinite journeys. Industries might reach some temporary, relatively stable pattern, but this is likely to be short lived. Endogenous change, due to corporate decisions to enter or exit the market or to develop new technologies, shifts the fitness landscape and the attractors in a system. Industries are subsystems of larger economic and social structures, which themselves are complex dynamic systems unfolding in unpredictable ways. Stacey (1995) relates this coevolution to Giddens's (1984) concept of structuration, in which the decisions and actions of agents change institutions, and these institutions in turn constrain and condition the behavior of individual agents.

Formulating a long-term plan is clearly a key strategic task facing any organization. People involved in planning have always known that models are simplified representations, that forecasts are uncertain, and that uncertainty grows over time. Nevertheless, our conventional understanding of linear models suggests that better models and a more accurate specification of starting conditions would yield better forecasts, useful for months if not years into the future. Complexity theory suggests otherwise; the payoff in terms of better forecasts from building more complex and more accurate models may be small. Meteorologists can improve their models by using more terrestial observations and dividing the atmosphere into a great many small interacting units of analysis; despite the application of successively more powerful computers, the accuracy of forecasts still falls off very rapidly after 3 or 4 days. Similarly, we cannot learn much about the future by studying the past: If history is the sum of complex and nonlinear interactions among people and nations, then history does not repeat itself. Concerning urban planning, Cartwright (1991) has noted that we have to acknowledge that "a complete understanding of some of the things we plan may be beyond all possibility." Stacey (1996:187) concluded that though short-term behavior might be predictable, "members of an organization, no matter how intelligent and powerful, will be unable to predict the specific long-term outcomes of their actions."

How long is the long term? Many discussions of complexity avoid this critical issue. For the weather, it is clear that more than 5 days is long-term forecasting. For biological evolutionary systems, the time frame might be millions of years. For firms and industries, the relationship between uncertainty and time is less clear; businesses have traditionally developed strategic plans for 3 or 5 years, though many companies also attempt to predict major technological trends over 10- and 20-year horizons.

\section{B. Dramatic Change Can Occur Unexpectedly}

Traditional paradigms of economics and strategy, built upon simplified assumptions of cause and effect, would suggest that small changes in parameters should lead to correspondingly small changes in the equilibrium outcome. Complexity theory forces us to reconsider this conclusion. Large fluctuations can be generated internally by deterministic chaotic systems, and small perturbations to networks, even when in the ordered state, can sometimes have major effects. Just as population levels of a species can crash as a result of 
ecosystem dynamics, economic systems can enter depressions and stock markets can crash even in the absence of external shocks.

The size of fluctuations from one period to the next in chaotic systems has a characteristic power law probability distribution. Under this distribution, large fluctuations occur more frequently than under the normal distribution, suggesting that managers might underestimate the potential for large changes in industry conditions or competitors' behavior. Small exogenous disturbances to complex systems can also cause unexpectedly large changes. The implication for business strategy is that the entry of one new competitor or the development of a seemingly minor technology can have a substantial impact on competition in an industry. The development of transistors, for example, originally appeared to be merely an incremental improvement over vacuum tubes. Similarly, the deal between IBM and Microsoft in 1980 to develop an operating system for their personal computer has profoundly shaped the software and hardware market since that time.

\section{Complex Systems Exhibit Patterns and Short-Term Predictability}

Social scientists are generally more interested in the order than the randomness of complex systems. Short-term forecasting is possible in a chaotic deterministic system because, given a reasonable specification of conditions at one time period, we can calculate the conditions the next time period. Again, the issue of time scales and usefulness arises. Weather forecasts based on sophisticated computer models using measurements from thousands of points around the globe do provide useful forecasts for a few days, and that is usually sufficient for purposes such as hurricane or flood warnings. How accurately must an industry model be specified for it to be useful over a 3-year period? We simply do not know, and there is clearly a need for more research in this area.

Strange attractors also give chaotic systems a degree of order and patterned behavior. Outcome variables such as prices or output fluctuate within certain bounds that are determined by the structure of the system and its parameters but not its initial conditions. In the context of business strategy, tendencies toward monopolistic behavior might be checked by negative feedback mechanisms, such as the entrance of new firms or antitrust action by the government. Similarly, oligopolistic industries such as the airline industry undergo alternating periods of price stability and price wars. Stacey (1996) described modeling efforts that demonstrate the emergence of predictable types of strategies in computer simulations of competition, such as predator, symbiosis, and parasite. Observing patterns is especially useful if we can associate different phases of the system with other characteristics; for example, there is a strong relationship between business cycles and other variables such as demand, interest rates, vendor lead times, and labor market conditions. Strategic planning can thus be focused on a limited number of scenarios defined by the system's attractors.

Chaotic systems can generate fractal patterns that are independent of scale; in other words, similar patterns are traced by a system whatever horizon is used to view it. Many natural phenomena, including ice crystals and tree branching, display this feature of recurrent patterns on different scales. Economic time series also appear to exhibit this property. Stock prices, for example, present a remarkably similar pattern whether one observes daily changes over a year or minute-by-minute changes over a day. The implications for business strategy are not entirely clear. One interpretation is that similar patterns of behavior might be expected whether one examines competition between countries. between firms in an industry, or even between departments in a firm. 


\section{Organizations Can Be Tuned to Be More Innovative and Adaptive}

Rather than expend large amounts of resources on forecasting for unpredictable futures, many writers have suggested that businesses emphasize flexibility, creativity, and innovation in response to the vagaries of the marketplace. The idea that organic structures are more effective than mechanistic ones in coping with turbulent environments does, of course, have a long pedigree in management studies (Burns and Stalker 1961). Complexity theory suggests that organic networks poised on "the edge of chaos" might give rise to self-organization and emergent order that enable firms to prosper in an era of rapid change (Allen 1988; Brown and Eisenhardt 1997). Advocates of this approach tend to invoke complexity in a metaphorical manner to lend the legitimacy of this new science to concepts that already have gained some discursive currency in management thinking. Stacey (1996:188) suggests that under conditions of complexity, "managers still need strategic plans, but they relate not to outcomes and actions to achieve them, but to methods of managing anxiety, power, difference, and connectivity."

Structuring organizations as decentralized, nonhierarchical networks is an important component of achieving this vision (Nohria and Eccles 1992; Senge, 1990; Stacey 1995; Wheatley 1996). The effectiveness of decentralization in promoting adaptation is suggested by Kauffman's (1993) work on network patches. The hastening of adaptation through a process of annealing, or jolting, could find its organizational analogy in bold forms of experimentation. Stacey (1995) argues that organizations can be tuned to the edge of chaos, where adaptation and creativity are maximized, by finding a balance between centralization and decentralization, between rigidity and disorder, though elsewhere Stacey (1993) asserts that the speed of information flow within an organization also serves this purpose. Lane and Maxfield (1996) have proposed that firms can enhance the process of variation and adaptation by seeking to build a web of external "generative" relationships, such as joint ventures with other firms, and allocating organizational resources to the more promising ventures. Organizational identity, culture, mission, and values can play a powerful role in integrating a decentralized, network form of organization, and some writers have suggested the metaphor of organizational mission and core values serving as an attractor (Mintzberg et al. 1996; Pascale 1990; Stacey 1995; Wheatley 1996).

Senge's work (1990) stresses the creativity and learning capacity of network organizations that encourage all workers to engage in a dialogue, "the free and creative exploration of complex and subtle issues, a deep 'listening' to one another and suspending of one's own views." Senge's work frequently invokes the metaphor of organizations as brains (Morgan 1997). Interest in the brain as a neural network has been growing. In a neurophysical study of magnetic fields generated by the brains of human subjects performing manual coordination tasks, Kelso and Fuchs (1995:64) concluded, "The brain is fundamentally a pattern forming, self-organized system governed by non-linear dynamical laws. ... The brain is a complex dynamical system in which, under certain conditions, spatiotemporal patterns emerge spontaneously and sustain themselves in a relatively autonomous fashion. ... It is poised on the brink of instability where it can switch flexibly and quickly."

The contention that complex organizations can be tuned to become more creative and adaptive is not without its problems. It is far from clear what it really means to say that an organization is in the ordered or chaotic regime. Knowing when it is on the edge of chaos seems a rather daunting prospect, though not as difficult perhaps as adjusting organizational parameters to reach that heady state. A fundamental issue is the nature and applica- 
tion of metaphors: Are they useful in communicating abstract concepts and complex data and serving as guides to action (Hill and Levenhagen 1995), or are they simply discursive devices used to legitimize and advocate particular organizational structures and processes? The notion of mission as an attractor is a case in point. Complexity theory suggests that attractors are emergent phenomena rather than an imposed constraint; moreover, dynamic systems traverse a substantial phase space around an attractor, which may represent multiple organizational states far from the mission.

The advocacy of shared values and mission to integrate an organization, though couched in language of organizational effectiveness, is not politically neutral (Alvesson and Deetz 1996). Asserting the commonality of interests of all organizational stakeholders and employees can be seen as an effort to articulate the particular interests of senior management as the general interest. Wrapped up in the discourse of empowerment and nonhierarchical networks, these visions have an obvious appeal for those concerned with organizational power relations. At the same time, this approach tends to obscure and mask sources of structural power that are embedded in broader social and economic systems and that are reproduced, like fractals, within industries, firms, and departments. Senge's work suffers a similar problem. His concept of free dialogue resonates with Habermas's (1984) theory of communicative action but lacks the sophisticated analysis of systematic barriers to such dialogue that Habermas provides.

\section{CONCLUSION}

This chapter has provided a basic description of complexity, distinguishing between chaos theory and network analysis. Dynamic nonlinear systems with feedback mechanisms can exhibit complex, unpredictable behavior within which underlying patterns and structure can be discerned. A working knowledge of the relevant theory and terminology of complexity is essential to enable readers to make their own judgments concerning the application of complexity to social science in general and strategy in particular.

It is important to acknowledge that complexity cannot simply be imported from the natural sciences and applied "off-the-shelf" to industries and firms. Future work needs to develop the concepts and analytical methods to take account of fundamental differences between social and natural sciences, relating to the nature of uncertainty in the social world; the degree of complexity entailed in multiple interlocking economic, social, political, and economic systems; and the role of human agency.

Complexity theory is not a complete break from traditional organization theory and scientific methods, in that it can be seen as a continuation and deepening of systems and behavioral approaches to organization theory. At another level, complexity represents a more fundamental shift in ontological and epistemological frames of reference. In dynamic systems, we seek webs of causation rather than simple linear relationships and accept the inherent complexity of economic systems rather than rely on traditional reductionist frameworks.

A growing number of empirical studies illustrate possible avenues for applying chaos theory and network analysis to studies of industries and business strategy. One stream of this research employs highly sophisticated computer models in what are effectively simulated laboratory experiments, while others are applying mathematical tests to discover whether outcome variables are indeed chaotic or simply random. Some researchers employ real human subjects to simulate interactions among firms or sectors of the economy. 
At the very least, theoretical and empirical work on complexity yields a few generalizable conclusions about the likely properties of competitive industries and some implications for strategy. The first and perhaps most important is the difficulty of long-term prediction and the possibility of sudden change, even in the absence of major external shocks. Closely related is the acceptance that industries will not reach a stable equilibrium. Under such conditions, the value of detailed strategic plans is questionable, and a more appropriate strategic stance would be preparation for multiple scenarios and the capability to adapt rapidly to changing conditions. An important question for future research is the meaning of long-term: It is possible that many industries will remain quite stable over a 5- or 10-year horizon, and that industries currently in a turbulent state will become more stable as technologies and markets mature. Planning for 20 or 50 years down the road may be a waste of time, but this is hardly news for corporate strategists.

Complexity does show significant promise in furthering our understanding of processes by which organizations adapt to their environments. Simulations suggest that networks can be tuned to adapt more readily when they are in the ordered regime but approaching the transition to chaos. Similarly, a number of procedures have been demonstrated to facilitate the adaptation process, such as simulated annealing and optimization for local patches. Applying these concepts in real firms is again not so easy; what does the boundary between order and chaos look like in an organization, and how does one tune the firm to get there? One approach is to employ complexity theory in a more qualitative or metaphorical way; for example, empowering employees to work individually and independently within the unifying structure of a common mission, culture, and intense communication is claimed to generate creativity, flexibility, and learning. Much of this work, however, appears to advocate for managerial concepts that were in vogue long before management consultants had heard of complexity.

A central question that needs to be resolved concerns the value of developing ever more detailed and sophisticated models. It is conceivable to construct a model of a particular industry with thousands of actors and a vast number of relationships specified among these actors. What would such a model be useful for? It might provide explanatory and predictive power for the short term, but the impact of uncertainty concerning future decisions by other human agents is likely to make such models much less reliable than our current weather forecasts, which are at least fundamentally deterministic. If each industry requires its own detailed model, this simulation approach might provide little inductive insight or generalizable theory that could not be gained by studying the industries themselves. It is possible that the modeling approach is missing the central insight concerning complex dynamic systems, that they cannot be adequately represented in parsimonious form. The only way to observe the future, aside from building a time machine, is to wait and watch the real system itself unfold.

Rather than attempt to divine the future, searching for deep structure and patterned behavior is probably a more useful avenue of inquiry. Complex systems contain attractors that signal a degree of underlying order. The relationship between a system's parameters and its attractors might provide some useful insights into industry behavior and firm strategy. Translating simulated patterns and attractors into organizational and strategic terms is not a simple proposition, however. Knowing the threshold values of parameters beyond which a system flips from one state to another could be one useful application. It must be remembered, however, that attractors are emergent phenomena, so one cannot construct a system to produce a given set of patterns.

Complexity theory offers promise and limitations similar to those presented by the advent of behavioral theories of the firm in an earlier era of organization studies. It provides 
many insights that are intuitively appealing to social scientists because they appear to reflect aspects of social and economic phenomena that cannot be captured in traditional rational actor models. The behavioral approach provides us with a deeper understanding of organizational processes, but it suffers from a lack of analytical tractability and predictive power. In a similar fashion, we may wonder whether complexity is capable of going beyond some useful insights and opening a rich new vein of organizational theory and analysis. A number of efforts have been made in this direction, using computer simulation techniques as well as qualitative methods. Results so far have been suggestive, but we are still far from discovering the secrets of organizational adaptation, learning, and creativity. A great deal more theoretical and empirical work is needed to reveal whether this is a mere intellectual curiosity or the highway to a fundamental understanding of the richness of structure, order, and uncertainty in complex economic and social phenomena.

\section{ENDNOTES}

1. See also special issues of Journal of Economic Theory, 40(1), 1986, and Journal of Economic Behavior and Organization, 8(3), 1987.

2. See Gleick (1987) for a good nontechnical overview.

3. The weather, of course, is subject to powerful influences by earth's annual orbit, which assure that the primary weather patterns are periodic, though nonperiodic patterns are superimposed upon this.

4. Recent evidence indicates that the earth's climate snaps into and out of ice ages in a matter of decades rather than centuries, raising concern about potentially irreversible catastrophic climate changes due to human emissions of greenhouse gases.

5. Kauffman (1995) is a more accessible work. Waldrop (1992) gives a good nontechnical overview of the complexity field, set in the intellectual ferment of the Santa Fe Institute. Other works by Sante Fe scholars include Gell-Mann (1994) and Holland (1995).

6. The $P$ bias parameter for a particular node measures the overall probability that it will be "on" given all the potential states of the nodes to which it is connected. The node is most "unstable" when $P=0.5$, and relatively stable when $P$ approaches 1 or 0 .

7. The degree of correlation is termed the epistatic coupling of the genes.

8. For examples of postmodern approaches to management and strategy, see Hassard and Parker (1993) and Knights and Morgan (1991).

9. Kelsey (1988) suggests that the influence of external systems can be modeled probabilistically, departing from the strict determinism of chaos. Levy (1994) employs this approach in his study of a supply chain.

\section{REFERENCES}

Abernathy, W. J., and Clark, K. B. (1985). Innovation: Mapping the winds of creative destruction. Research Policy, 14: 3-22.

Allen, P. M. (1988). Dynamic models of evolving systems, System Dynamics Review, 4: 109-130.

Allen, P., Clark, N., and Perez-Trajo, F. (1992). Strategic planning of complex economic systems, Review of Political Economy, 4(3), 275-290.

Alvesson, M., and Deetz, S. (1996). Critical theory and postmodernism approaches to organizational studies, Handbook of Organization Studies (S. R. Clegg, C. Hardy, W. R. Nord, eds.), Sage, Thousand Oaks, California.

Anderson, P., and Tushman, M. L. (1990). Technological discontinuities and dominant designs: A cyclical model of technological change, Administrative Science Quarterly, 35: 604-633. 
Arthur, W. B. (1989). Competing technologies, increasing returns, and lock-in by historical events, The Economic Journal: 99. 116-131.

Arthur, W. B. (1996). Increasing returns and the new world of business, Harvard Business Review July-August, 100-109.

Bak, P., and Chen, K. (1991). Self-organized criticality, Scientific American, 264( I): 46-53.

Bartlett, A. and Ghoshal, S. (1989). Managing Across Borders: The Transnational Solution, Harvard Business School Press, Boston.

Baumol, W., and Benhabib, J. (1989). Chaos: Significance, mechanism, and economic applications, Journal of Economic Perspectives, 3: 77-105.

Brock, W., and Malliaris, A. (1989). Differential Equations, Stability and Chaos in Dynamic Systems, North-Holland, New York.

Brown, S., and Eisenhardt, K. (1997). The art of continous change: Linking complexity theory and time-paced evolution in relentlessly shifting organizations, Administrative Science Quarterly, 42: 1-34.

Burns, T., and Stalker, G. M. (1961). The Management of Innovation, Tavistock, London.

Butler, A. (1990). A methodological approach to chaos: Are economists missing the point? Federal Reserve Bank of St. Louis, 72 (13): 36-48.

Camerer, C. F. (1991). Does strategy research need game theory? Strategic Management Journal, 12: 137-153.

Cartwright, T. J. (1991). Planning and chaos theory, Journal of the American Planning Association, 57(1): 44-56.

Cyert, R. M., and March, J. G. (1963). A behavioral theory of the firm, Prentice Hall, Englewood Cliffs, New Jersey.

Feigenbaum, M. J. (1983). Universal behavior in nonlinear systems, Physica, 7: 16-39.

Ford, J. (1983). How random is a coin toss? Physics Today, 36: 40-47.

Gell-Mann. M. (1994). The Quark and the Jaguar: Adventures in the Simple and the Complex, W. H. Freeman, New York.

Ghemawat, P. (1997). Games Businesses Play: Cases and Models, MIT Press, Cambridge, Massachusetts.

Giddens, A. (1984). The Constitution of Society: Outline of the Theory of Structuration, University of California Press, Berkeley, California.

Gleick, J. (1987). Chaos, Penguin Books, New York.

Goldberger, A. L., Rigney, D. R., and West, B. J. (1990). Chaos and fractals in physiology, Scientific American, 263: 43-49.

Habermas, J. (1984). The Theory of Communicative Action, Polity Press, Cambridge, Massachusetts.

Hannan, M., and Freeman, J. (1984). Structural inertia and organizational change, American Sociological Review, 82: 929-964.

Hassard, J., and Parker, M.. ed. (1993). Postmodernism and Organizations, Sage, Newbury Park, California.

Haveman. H. (1992). Between a rock and a hard place: Organizational change and performance under conditions of fundamental environmental transformation, Administrative Science Quarterly, 37: 48-75.

Henderson, R., and Clark, K. B. (1990). Architectural innovation: The reconfiguration of existing product technologies and the failure of established firms, Administrative Science Quarterly, 35: 9-30.

Hill, R. C., and Levenhagen. M. (1995). Metaphors and mental models: Sensemaking and sensegiving in innovative and entrepreneurial activities, Journal of Management, 21(6): 1057-1074.

Holland, J. (1995). Hidden Order: How Adaptation Builds Complexity, Addison-Wesley, Reading, Massachusetts.

Hsieh, D. A. (1991). Chaos and nonlinear dynamics: Application to financial markets, Journal of Finance, 46(5):1839-1877.

Katz. D., and Kahn, R. L. (1966). The Social Psychology of Organizations, John Wiley, New York.

Kauffman, S. A. (1993). The Origins of Order: Self-Organization and Selection in Evolution, Oxford University Press, Oxford. 
Kauffman, S. A. (1995). At Home in the Universe: The Search for the Law's of Self-Organization and Complexity, Oxford University Press. Oxford.

Kelsey, D. (1988). The economics of chaos or the chaos of economics, Oxford Economic Papers, 40: $1-31$.

Kelso, J. A., and Fuchs, A. (1995). Self-organizing dynamics of the human brain: Critical instabilities and Sil'nikov chaos, Chaos, 5(1):64-69.

Kiel, D., and Elliott, E. (1996). Chaos Theory and Social Sciences (D. Kiel and E. Elliott, eds.), University of Michigan Press, Ann Arbor.

Knights, D., and Morgan, G. (1991). Corporate strategy, organizations, and subjectivity: A critique, Organization Studies, 12(2):251-273.

Lane, D., and Maxfield, R. (1996). Strategy under complexity: Fostering generative relationships, Long Range Planning, 29(2):215-231.

Langton, C. G. (1989). Artificial life, Santa Fe Institute Studies in the Sciences of Complexity; Vol. 6, Addison Wesley, Redwood City, California.

Levinthal, D. (1997). Adaptation on rugged landscapes, Management Science, 43(7):934-950.

Levy, D. L. (1994). Chaos theory and strategy: Theory, application, and managerial implications, Strategic Management Journal, 15: 167-178.

Lorenz, E. N. (1963). Deterministic non-periodic flow, Journal of the Atmospheric Sciences, 20 : $130-141$.

Mayer-Kress, G., and Grossman, S. (1989). Chaos in the international arms race, Nature, 337: 701-704.

Medio, A. (1991). Continuous time models of chaos in economics, Journal of Economic Behavior and Organization, 16: 115-152.

Merry, U. (1995). Coping with Uncertainty: Insights from the New Sciences of Chaos, Self-Organization, and Complexity, Praeger, Westport, Connecticut.

Mintzberg, H., Pascale, R., Goold, M., and Rumelt, R. (1996). The 'Honda' effect revisited, California Management Review, 38(4):78-117.

Mirowski, P. (1990). From Mandelbrot to chaos in economic theory, Southern Economic Journal, 57: 289-318.

Morgan, G. (1997). Images of Organization, 2nd ed., Sage, Thousand Oaks, California.

Mosekilde, E., and Rasmussen, S. (1986). Technical economic succession and the economic long wave, European Journal of Operational Research, 25: 27-38.

Nohria, N., and Eccles, R. G. (1992). Networks and Organizations, Harvard Business School Press, Boston.

Pascale, R. (1990). Managing on the Edge: How the Smartest Companies Use Conflict to Stay Ahead, Simon \& Schuster, New York.

Porter, M. E. (1990). The Competitive Advantage of Nations, Free Press, New York.

Radzicki, M. J. (1990). Institutional dynamics, deterministic chaos, and self-organizing systems, Journal of Economic Issues, 24(1):57-102.

Senge, P. M. (1990). The Fifth Discipline: The Art and Practice of the Learning Organization, Doubleday, New York.

Shackley, S., Wynne, B., and Waterton, C. (1996). Imagine complexity: The past, present and future potential of complex thinking, Futures, 28(3):201-225.

Simon, H. A. (1957). Administrative Behavior, 2nd ed., Macmillan, New York.

Simon, H. A. (1962). The architecture of complexity, Proceedings of the American Philosophical Societr, 106: 467-482.

Simon, H. A. (1964). Approaching the theory of management. Toward a Unified Theory of Management, (H. Koontz ed.), McGraw Hill, New York.

Singh, J., House, R., and Tucker, D. (1986). Organizational change and organizational mortality, Administrative Science Quarterly, 31: 587-611.

Stacey, R. (1993). Strategy as order emerging from chaos, Long Range Planning, 26(1):10-17.

Stacey, R. (1995). The science of complexity: An alternative perspective for strategic change processes, Strategic Management Journal, 16: 477-495. 
Stacey, R. (1996). Emerging strategies for a chaotic environment. Long Range Planning, 29(2):182-189.

Sterman, J. D. (1989). Deterministic chaos in an experimental economic system, Journal of Economic Behavior and Organization, 12: 1-28.

Stewart, I. (1989). Does God play dice? Oxford University Press, Oxford.

Thompson, J. D. (1967). Organizations in Action: Social Science Bases of Administrative theory, McGraw-Hill, New York.

Tushman, M. L., and Anderson, P. (1986). Technological discontinuities and organizational environments, Administrative Science Quarterly, 31: 439-465.

Waldrop, M. M. (1992). Complexity: The Emerging Science at the Edge of Order and Chaos, Touchstone, New York.

Wheatley, M. (1992). Leadership and the New Science, Berrett-Koehler, San Francisco.

Wheatley, M. K., M. (1996). A Simpler Way, Berrett-Koehler, San Francisco.

Womack, J. P., Jones, D. T., and Roos, D. (1990). The Machine That Changed the World, Rawson Macmillan, New York. 